\title{
Assessment of recombinant plasmid expressing fusion antigen Ag85B-Rv3425 in management of acute tuberculosis infection in mice
}

\author{
HUICHANG HUANG $^{1 *}$, FEIFEI WANG $^{2 *}$, ENZHUO YANG $^{1}$, \\ HONGHAI WANG ${ }^{3}$, PENG GAO $^{4}$ and HONGBO SHEN ${ }^{1}$ \\ ${ }^{1}$ Unit of Anti-Tuberculosis Immunity, CAS Key Laboratory of Molecular Virology and Immunology, \\ Institute Pasteur of Shanghai, Chinese Academy of Sciences, Shanghai 200031; ${ }^{2}$ Department of \\ Medical Microbiology and Parasitology, Shanghai Medical College, Fudan University, Shanghai 200032; \\ ${ }^{3}$ State Key Laboratory of Genetic Engineering, School of Life Sciences, Fudan University, Shanghai 200433; \\ ${ }^{4}$ Department of Urology, Huashan Hospital, Fudan University, Shanghai 200040, P.R. China
}

Received August 17, 2017; Accepted January 4, 2018

DOI: $10.3892 /$ etm.2018.5785

\begin{abstract}
The emergence of drug-resistant tuberculosis (TB) and HIV-TB co-infection fuels an urgent need to develop novel therapeutic approaches, including therapeutic vaccines. Therapeutic vaccines have been proven to be a good strategy by inducing antigen specific immune responses against $\mathrm{TB}$ infection. In the present study, a recombinant plasmid based on lentiviral vector expressing fusion antigen Ag85B-Rv3425 (A3), and was constructed the immunogenicity and treatment effects in TB mice were assessed. The results showed that A3 delivered by the plasmid could be expressed appropriately in vivo and induced higher production of tumor necrosis factor- $\alpha$ and interleukin- 2 compared with A3 recombinant protein in mice. Moreover, the recombinant plasmid expressing A3 confered resistance to acute $\mathrm{TB}$ infection in mice, characterized by a reduction in the bacterial load in the lungs and spleen, as well as attenuated TB lesions in lung tissues. These results implicated that the recombinant plasmid based
\end{abstract}

Correspondence to: Professor Hongbo Shen, Unit of Anti-Tuberculosis Immunity, CAS Key Laboratory of Molecular Virology and Immunology, Institute Pasteur of Shanghai, Chinese Academy of Sciences, 320 Yueyang Road, Shanghai 200031, P.R. China

E-mail: hbshen@ips.ac.cn

Professor Peng Gao, Department of Urology, Huashan Hospital, Fudan University, 12 Wulumuqi Zhong Lu Road, Shanghai 200040, P.R. China

E-mail: gaopeng76@163.com

${ }^{*}$ Contributed equally

Key words: lentiviral vector, plasmid, Ag85B, therapeutic vaccine, tuberculosis on lentiviral vector expressing A3 is a potent and promising therapeutic agent to treat acute $\mathrm{TB}$ infection.

\section{Introduction}

Tuberculosis (TB), a worldwide infectious disease caused by Mycobacterium tuberculosis (Mtb), continues to be a major threat to human health $(1,2)$. Unfortunately, BCG (bacillus Calmette-Guérin), the only vaccine widely used against TB, provides varied protective efficacy $(0-80 \%$ in randomized control trials) (3-5). Recently, the emergence of drug-resistant Mtb stains as well as HIV-TB co-infection poses numerous complexities for TB control (6,7). More effective vaccines and more potent immune strategies against TB are now in urgent need.

Therapeutic vaccines shed light on TB control by inducing antigen specific immune responses against Mtb in vivo (8), and Mtb potent antigen encoding genes are delivered into host cells via plasmid, adenovirus or lentivirus for in vivo gene expression $(9,10)$. As a novel immunotherapy strategy, therapeutic vaccine has been successful for TB control not only in latent TB infection model but also in acute infection model $(11,12)$.

Ag85B-Rv3425 (A3) is a fusion protein of Ag85B and Rv3425, a member of the PE (Pro-Glu) and PPE (Pro-Pro-Glu) family, located in $\mathrm{RD} 11$ region which is absent in $\mathrm{BCG}$ strains $(13,14)$. It has been reported rBCG::Ag85B-Rv3425 vaccine, formed by co-expressing Rv3425 and Ag85B in $\mathrm{BCG}$, provides better protective efficacy against Mtb challenge compared with BCG (15). Recently, it was reported that A3 delivered into mice via lentivirus with one single dose administration confers post-infection resistance to acute TB infection (11).

In this study, we constructed a recombinant plasmid based on lentiviral vector expressing fusion antigen Ag85B-Rv3425 (A3) and assessed its immunogenicity and treatment effect in TB mice. We found that in vivo expression of A3 could induce more IL-2 as well as TNF- $\alpha$ compared with immunization using A3 purified from $E$. coli, which is recombinant fusion 
protein of Ag85B-Rv3425 purified from recombinant E. coli. Moreover, in vivo expression of $\mathrm{A} 3$ provides immunity to acute Mtb infection characterized by reduced Mtb burden in lung and spleen and attenuated pathology in lung tissue.

\section{Materials and methods}

Animals. A total of 6-8 week-old female C57BL/6 mice were purchased from SLAC Laboratory Animal Center (Shanghai, China). All mice were maintained under specific-pathogen-free (SPF) conditions in animal facilities at Animal Biosafety Level (ABSL)-III lab of Wuhan University and given sterile water, mouse chow and bedding. All mice experiments were performed in accordance with recommendations in the Wuhan University Research Council Guide for Care and Use of Laboratory Animals. Animal study protocols were also reviewed and approved by the Wuhan University Institutional Animal Care and Use Committee.

Plasmid and proteins. pLenti 6.3 plasmid was purchased from Biomiga (San Diego, CA, USA). The cloning of ag85b-rv3425 fusion gene into pLenti6.3 plasmid was our described previously (11). A3 protein were purified as our described previously (13). Endotoxins concentration of A3 protein was tested using the commercially available Quantitative Chromogenic End-point Tachypleus Amebocyte Lysate reactivity endotoxin kit (Chinese Horseshoe Crab Reagent Manufactory, Xiamen, China). A total of $<5.0 \mathrm{EU} / \mathrm{mg}$ endotoxins was observed and purified A3 protein was subsequently subjected to animal immunization.

Immunization. C57BL/6 mice were divided into four groups (20 mice per group). At week 0, mice in PBS group received a subcutaneous injection of empty plasmids in PBS and mice in A3-Pro group were injected subcutaneously with $50 \mu \mathrm{g}$ recombinant A3 protein in $250 \mu \mathrm{g}$ dimethyl dioctadecyl-ammonium bromide (DDA) adjuvant (Sigma). Mice in A3-Vec group were immunized by an intramuscular injection of $50 \mu \mathrm{g}$ recombinant pLenti6.3 plasmid harboring ag85b-rv3425 fusion gene. Mice in Vec group were immunized by an intramuscular injection of $50 \mu \mathrm{g}$ pLenti6.3 empty plasmid vector. All mice received 3 injections at a two-week interval. 4 weeks after the last injection, mice were sacrificed for immune response evaluation ( 8 mice per group).

Separation of spleen lymphocytes. Spleen from each mouse was collected under aseptic condition immediately after sacrifice. The single-cell suspension $(4 \mathrm{ml})$ was obtained by gently grounding spleen through a $75 \mu \mathrm{m}$ cell strainer and underlayed by $4 \mathrm{ml}$ pre-warmed Lymphocyte-M (Cedar Lane Lab, Burlington, VT, USA). Density-gradient centrifugation was performed at $1,250 \mathrm{x} \mathrm{g}$ for $25 \mathrm{~min}$ to isolate splenocytes $(16,17)$. Cells were counted and diluted to a final concentration of $5 \times 10^{6}$ cells/ml in RPMI-1640 medium (Invitrogen; Thermo Fisher Scientific, Inc., Waltham, MA, USA) supplemented with $10 \mathrm{U} / \mathrm{ml}$ penicillin, $10 \mu \mathrm{g} / \mathrm{ml}$ streptomycin and $10 \%$ fetal bovine serum (Invitrogen; Thermo Fisher Scientific, Inc.).

Enzyme-linked ImmunoSpot (ELISPOT) assay. Filtration plates (96-well; Merck KGaA, Darmstadt, Germany) were coated with monoclonal antibodies against mouse TNF- $\alpha$ (U-CyTech, Utrecht, The Netherlands) for $16 \mathrm{~h}$ at $4^{\circ} \mathrm{C}$. After being washed with phosphate-buffered saline (PBS), wells were blocked with $200 \mu$ l blocking buffer $\mathrm{R}$ for $1 \mathrm{~h}$ at $37^{\circ} \mathrm{C}$. Spleen lymphocytes $(2.5 \times 105$ cells $)$ were mixed with $10 \mu \mathrm{g} / \mathrm{ml} \mathrm{A} 3$ protein and added to each well. Stimulation was completed by incubating cells at $37^{\circ} \mathrm{C}$ for $36 \mathrm{~h}$. After being washed with PBST for five times, each well was filled with $100 \mu \mathrm{l}$ of biotinylated detection antibodies, and incubated at $37^{\circ} \mathrm{C}$ for $1 \mathrm{~h}$. Then, wells were washed five times and incubated with $100 \mu \mathrm{l}$ of streptavidin-HRP for $1 \mathrm{~h}$ at $37^{\circ} \mathrm{C}$. After being washed, $100 \mu \mathrm{l}$ of AEC substrate solution was added to each well and plates were incubated for $25 \mathrm{~min}$ at room temperature in the dark. Color development was stopped by rinsing both sides of the polyvinylidene fluoride membrane in 96-well filtration plates with demineralized water. Finally, the plates were air dried and the amount of positive spots was counted by a dissecting microscope. The number of TNF- $\alpha$ expressing cell was calculated by spot forming units per million cells.

Cytokines assay. $2.5 \times 10^{6}$ cells were stimulated with $10 \mu \mathrm{g} / \mathrm{ml}$ A3 fusion protein at $37^{\circ} \mathrm{C}, 5 \% \mathrm{CO}_{2}$ and high humidity for $36 \mathrm{~h}$ as described before (18). The supernatant from stimulated spleen lymphocytes was collected, and the concentrations of TNF- $\alpha$ and IL-2 were measured by BD ${ }^{\mathrm{TM}}$ Cytometric Bead Array kit (BD Biosciences, Franklin Lakes, NJ, USA) according to the manual in the kit.

Mtb infection and immunotherapy. For immunotherapy, mice were infected intravenously via lateral caudal vein with $6.8 \times 10^{5}$ live Mtb strain H37Rv. At 4 weeks after infection, immunotherapy was applied and injection strategy was the same as immune responses evaluation listed above. At 4 weeks later at week 12, mice were sacrificed for colony-forming unit (CFU) counts (6 mice per group) and histopathological analysis in lung and spleen (6 mice per group).

Histopathology analysis. Each lung was excised and fixed in $4 \%$ neutral-buffered paraformaldehyde solution for $24 \mathrm{~h}$. Then lung tissue was embedded with paraffin. Series of sections with a thickness of 4-7 $\mu \mathrm{m}$ were then cut and stained with haematoxylin and eosin under standard methods. Double blind analysis was then made by board-certified pathologists and more than 10 slides of each lung were evaluated.

Statistical analysis. Statistical analysis was performed using SPSS Statistics 17.0 for Windows software package. Results were subjected to one-way Anova test followed by multi-comparison testing. $\mathrm{P}<0.05$ was considered to indicate a statistically significant difference.

\section{Results}

Fusion antigen A3 delivered by plasmid pLenti6.3 could be expressed in mice and induce immune response. To make fusion Mtb antigen A3 expressed appropriately in eukaryotic cells, we constructed a recombinant plasmid expressing A3 (A3-Vec) by cloning ag85b-rv3425 fusion gene fragment 

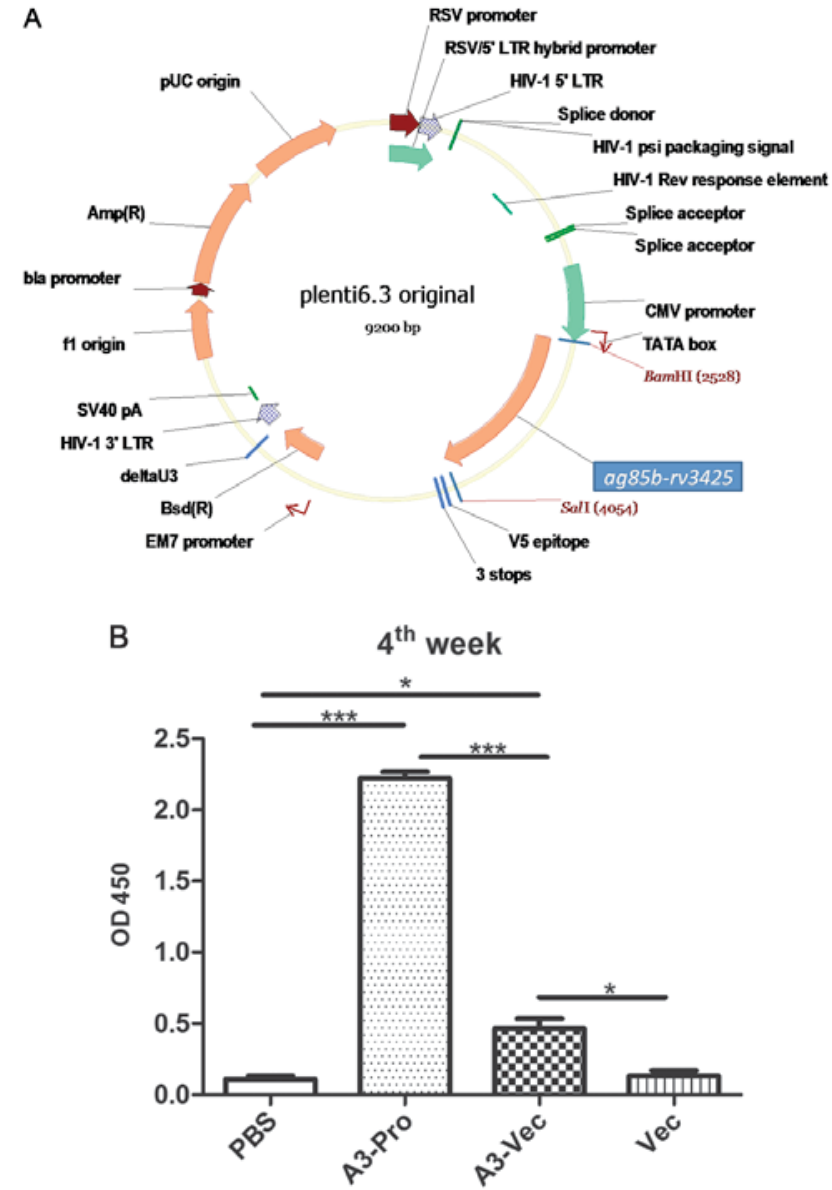

Figure 1. (A) A3 antigen specific antibody $\operatorname{IgG}$ response after immunization with recombinant pLenti6.3 plasmid harboring ag85b-rv3425 fusion gene. Mice were vaccinated with PBS, A3-Pro, A3-Vec and empty plasmid plenti6.3 plasmids (Vec). (B) 4 weeks after the last injection, plasma from each mouse was collected and titer of $\mathrm{A} 3$ specific IgG was evaluated via ELISA. ${ }^{*} \mathrm{P}<0.05,{ }^{* * *} \mathrm{P}<0.001$. Pro, protein; Vec, vector; IgG, immunoglobulin G; PBS, phosphate-buffered saline

and inserting it into pLenti6.3 vector, whose CMV promoter upstream could drive the transcription of ag85b-rv3425 fusion gene in eukaryotic cells. Then, $50 \mu \mathrm{g}$ endotoxin-free recombinant plasmids $(\mathrm{A} 3-\mathrm{Vec})$ were injected into mice for 3 times at a two-week interval. As controls, $50 \mu \mathrm{g}$ endotoxin-free empty plasmids (Vec) and $50 \mu \mathrm{g}$ recombinant proteins A3 (A3-Pro) purified from E. coli were also injected for 3 times at a two-week interval, respectively. In the 4th week after the last immunization, mice were sacrificed for immune response analysis. We have detected A3 fusion protein specific antibody $\mathrm{IgG}$ response in $\mathrm{A} 3-\mathrm{Vec}$ group and found that injection of recombinant plasmids A3-Vec, as well as recombinant proteins $\mathrm{A} 3$, induced $\mathrm{A} 3$ specific antibody Immunoglobulin $\mathrm{G}$ ( $\mathrm{IgG}$ ) production (Fig. 1). It indicates that Mtb fusion gene ag85b-rv3425 delivered by pLenti6.3 plasmid could be expressed and translated into fusion protein in mice and then recognized by immune system.

A3 delivered by pLenti6.3 plasmid increased the production of Th1-type cytokines. Next, we want to know whether A3 delivered by plasmid could induce protective immune response since it could be expressed in mice. As Th1-type
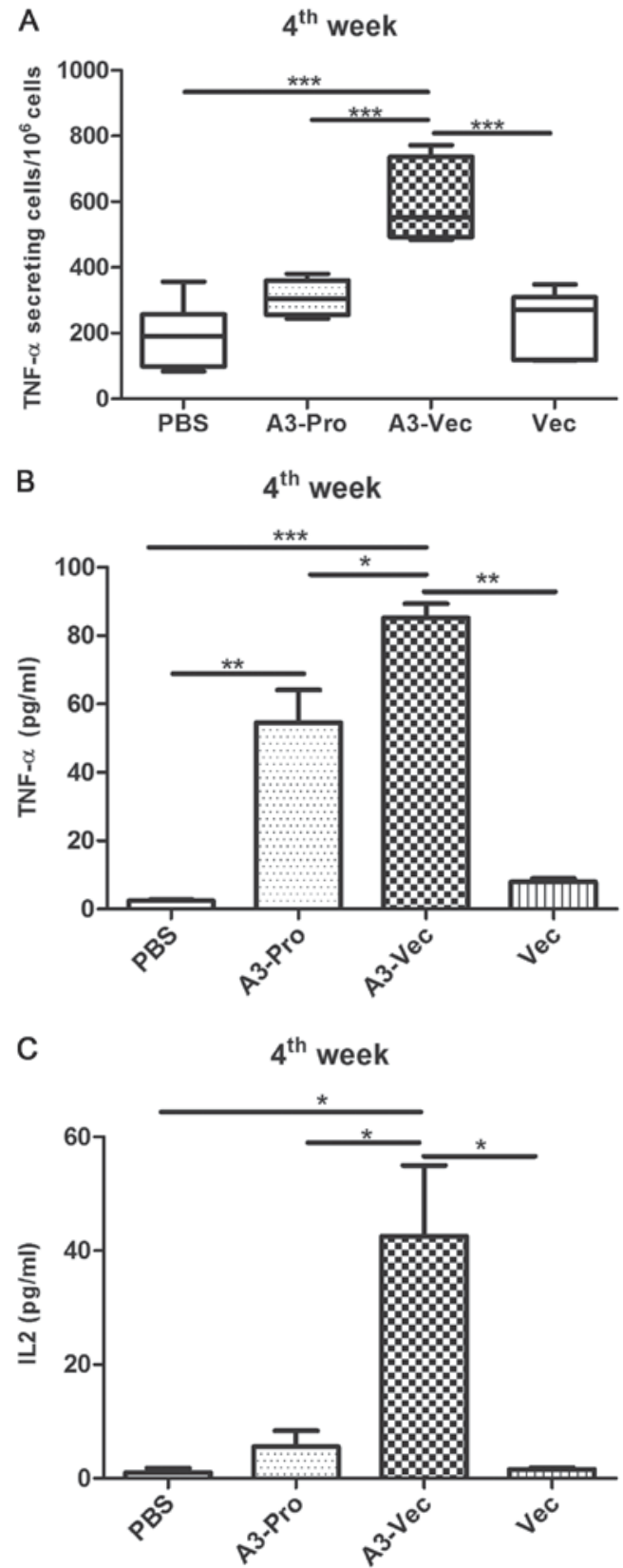

Figure 2. A3 delivered by pLenti6.3 vector elicits Th1-type immune responses. (A) ELISPOT assay was utilized to test the TNF- $\alpha$ producing ability of spleen lymphocytes upon A3 protein stimulation. After stimulation with A3 protein for $36 \mathrm{~h}$, culture supernatant from each mouse was subjected to $\mathrm{BD}^{\mathrm{TM}}$ Cytometric Bead Array to determine the concentration of (B) TNF- $\alpha$ and (C) IL-2. ${ }^{*} \mathrm{P}<0.05,{ }^{* *} \mathrm{P}<0.01,{ }^{* * *} \mathrm{P}<0.001$. Pro, protein; Vec, vector; TNF, tumour necrosis factor; IL, interleukin.

immune responses plays important role in protection against Mtb $(18,19)$. To identify whether A3-Vec plasmid could induce Mtb-specific Th1-type immune responses, we evaluated the production of Th1-type cytokines in mice. ELISPOT results show that there are more TNF- $\alpha$ producing cell in mice of A3-Vec group than those in A3-Pro group, Vec (empty plasmids) group and PBS group (Fig. 2A). And we found that the concentration of TNF- $\alpha$ in culture supernatant of spleen lymphocytes from A3-Vec immunized mice was higher than that from other groups determined with enzyme linked immunosorbent assay (ELISA) methods with recombinant A3 protein stimulation in vitro (Fig. 2B). 
Table I. Schedule of therapy experiments against Mtb challenge.

\begin{tabular}{|c|c|c|c|c|c|}
\hline Group & 0 week & 4th week & 6th week & 8th week & 12 th week \\
\hline PBS & Infection & PBS & PBS & PBS & Necropsy \\
\hline A3-Pro & Infection & A3-Pro & A3-Pro & A3-Pro & Necropsy \\
\hline A3-Vec & Infection & A3-Vec & A3-Vec & A3-Vec & Necropsy \\
\hline Vec & Infection & Vec & Vec & Vec & Necropsy \\
\hline
\end{tabular}

Pro, protein; Vec, vector; Mtb, Mycobacterium tuberculosis; PBS, phosphate-buffered saline.

A

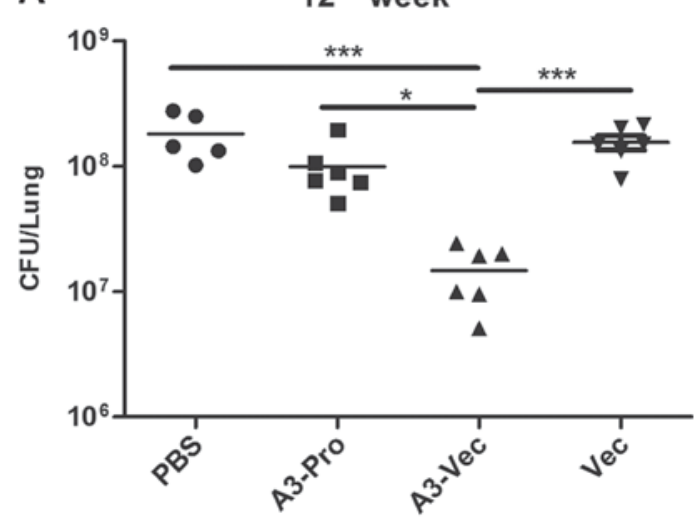

B
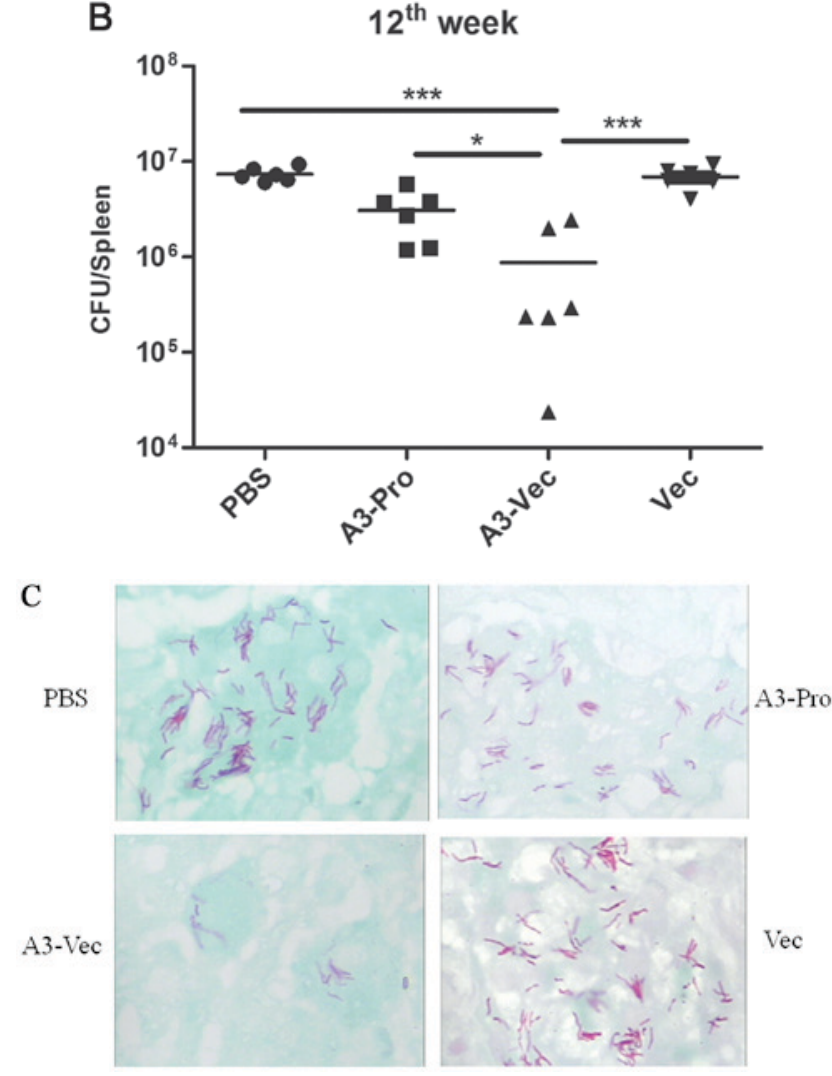

Figure 3. A3 delivered by pLenti6.3 vector restricts Mtb growth in mice in vivo. (A) Mean CFU counts of bacilli in lung homogenates. Lung tissue homogenates generated from each mouse was serially diluted and used for CFU enumeration. (B) Mean CFU counts of bacilli in spleen homogenates. Spleen tissue homogenates generated from each mouse was serially diluted and used for CFU enumeration. (C) Representative fast-acid staining image for lung tissue. The color of Mtb bacteria is purple. ${ }^{*} \mathrm{P}<0.05,{ }^{* * *} \mathrm{P}<0.001$. Magnification, x1,000. Pro, protein; Vec, vector; PBS, phosphate-buffered saline; CFU, colony-forming unit.

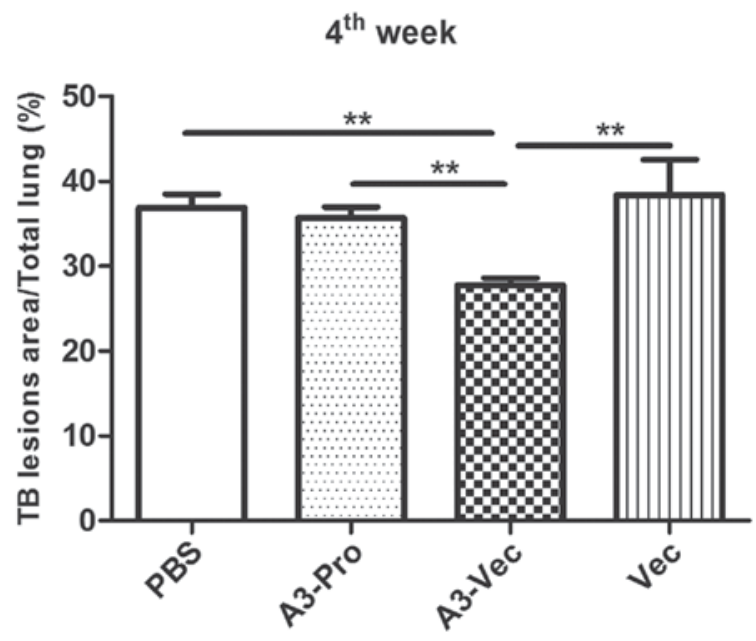

Figure 4. A3 delivered by pLenti6.3 vector exhibits immune resistance to TB lesions in lungs. The percentage of histopathological lesions area in total lungs. The area of histopathological lesions was calculated and compared with total lung area. ${ }^{* *} \mathrm{P}<0.01$. Pro, protein; Vec, vector; PBS, phosphate-buffered saline.

Moreover, we detected a much higher concentration of IL-2 production in spleen lymphocytes from A3-Vec group upon $\mathrm{A} 3$ protein stimulation (Fig. 2C). It seems that A3-Vec immunization exhibited higher ability in inducing Th1-type cytokines production than immunization of $\mathrm{A} 3$-proteins or empty plasmids.

We also compared the percentages/numbers of $\mathrm{T}$ cell subsets (CD3+, CD4+ and CD8+ T cells subsets) in spleen lymphocytes among these groups, and found no significant difference (data not shown).

Administration of A3-Vec plasmids effectively reduces $M t b$ burdens in lungs and spleens of Mtb infected mice. Since A3-Vec could induce antigen specific Th1-type cytokines production, we want to know whether A3-Vec could relieve symptoms of TB in mice. To address this, we evaluated the therapeutic effect of A3-Vec injection in Mtb-infected murine model. Mice were challenged by intravenous injection with Mtb H37Rv to establish acute infection. After 4 weeks of infection, we began to inject A3-Vec plasmids, A3-Pro, Vec (empty plasmids) and PBS control into TB mice according to the schedules listed in Table I. At the 12th week, mice were sacrificed, and bacteria burdens in lungs and spleens were counted. We found that there was a statistically significant CFU burden reduction in lung, as well as spleen, of A3-Vec 
treated mice comparing with Vec and PBS controls or even A3-Pro treated mice (Fig. 3A). And mice in A3-Vec present the lowest CFU counts in spleen (Fig. 3B). Acid-fast staining results showed that many Mtb bacteria aggregating were found in lung tissue from control groups (PBS and empty plasmid-Vec) as well as from A3-Pro treated group, while fewer Mtb bacteria were seen in slide from A3-Vec group (Fig. 3C). These results demonstrate that A3-Vec administration could provide immune protection to acute Mtb infection through inhibiting Mtb growth in vivo.

Administration of A3-Vec plasmids confers immune resistance to TB lesions. To identify whether administration of A3-Vec plasmid could decrease TB lesions in lungs caused by Mtb infection, we performed histopathological analysis to compare gross pathology in lungs of mice in different groups. TB lesions area percentage was calculated by double blind pathologists. We found that A3-Vec group exhibits the least lesion area among these four groups (Fig. 4). More detailed histopathological images illustrated mild lesion with small area of epithelioid cells and lymphocytes hyperplasia in A3-Vec group, while there were large area of lymphocytes, eosinophils and macrophages hyperplasia in pulmonary alveoli of the other three groups (data not shown). It means that administration of A3-Vec in mice conferred immune resistance to lesions caused by TB acute infection.

\section{Discussion}

The emergence of drug-resistant TB strains and HIV-TB co-infection makes global TB control a real challenge, it's in urgent need to facilitate worldwide control of TB (20). Immunotherapy is regarded as a potential approach to eliminate Mtb (21). In this study, we constructed a recombinant plasmid based on lentiviral vector expressing multiple antigens and assessed its immune response and treatment effect in TB mice.

As our previously reported, fusion protein Ag85B-Rv3425 is proved to be an effective multiple epitopes antigen $(11,13)$. In our study, we found that recombinant plasmid A3-Vec, like lentivirus delivered A3 (11), could induce A3 fusion protein specific antibody IgG response, indicating that it could be expressed in mice and then recognized by immune system. Moreover, we have observed immunization of plasmid A3-Vec could induce high Th1-type cytokines production, such as IL-2 and TNF- $\alpha$ (22), in spleen lymphocytes in A3-Vec treated mice. Moreover, recombinant plasmid A3-Vec shows potent effective therapeutic effect in acute Mtb infected mice. After immunotherapy, mice in A3-Vec group present the lower CFU counts in lung as well as in spleen than the control groups and exhibits significantly mild tuberculous lesions, which showed a symbol of epithelioid cells and lymphocytes hyperplasia combined with exudative inflammation of pneumonedema.

At the same time, we also compared the immunogenicity and treatment effects of the same fusion antigen A3 delivered by plasmid and recombinant protein purified from recombinant $E$. coli (subunit vaccine) in TB mice. We found that immunization of plasmid A3-Vec could induce higher Th1-type cytokines production, such as IL-2 and TNF- $\alpha$, than immunization of A3-Pro recombinant protein, though A3-Pro can induce high $\operatorname{IgG}$ in mice. And A3-Vec can decrease the CFU counts in lung as well as in spleen and alleviate tuberculous lesions in acute Mtb infected mice comparing to A3-Pro. It implies that plasmid vector might be a more effective method in antigen presentation than protein subunit vaccine.

In summary, our results show a good therapeutic effect of recombinant plasmid $\mathrm{A} 3-\mathrm{Vec}$ treatment against acute TB infection in mice. Further study might be conducted in non-human primates or even in humans to assess therapeutic effect of this recombinant plasmid.

\section{Acknowledgements}

This study was supported by the grants from the National Natural Science Foundation of China (no. 81401711), Nature Science Foundation of Shanghai Science and Technology Committee (no. 14ZR1444300).

\section{References}

1. Sulis G, Centis R, Sotgiu G, D'Ambrosio L, Pontali E, Spanevello A, Matteelli A, Zumla A and Migliori GB: Recent developments in the diagnosis and management of tuberculosis. NPJ Prim Care Respir Med 26: 16078, 2016.

2. Raviglione M and Sulis G: Tuberculosis 2015: Burden, challenges and strategy for control and elimination. Infect Dis Rep 8: $6570,2016$.

3. de Jonge MI, Brosch R, Brodin P, Demangel C and Cole ST: Tuberculosis: From genome to vaccine. Expert Rev Vaccines 4: 541-551, 2005.

4. Zhang L, Ru HW, Chen FZ, Jin CY, Sun RF, Fan XY, Guo M, Mai JT, Xu WX, Lin QX and Liu J: Variable virulence and efficacy of BCG vaccine strains in mice and correlation with genome polymorphisms. Mol Ther 24: 398-405, 2016.

5. Bali P, Tousif S, Das G and Van Kaer L: Strategies to improve BCG vaccine efficacy. Immunotherapy 7: 945-948, 2015.

6. Ahmed MM, Velayati AA and Mohammed SH: Epidemiology of multidrug-resistant, extensively drug resistant, and totally drug resistant tuberculosis in middle east countries. Int $\mathbf{J}$ Mycobacteriol 5: 249-256, 2016.

7. Odone A, Matteelli A, Chiesa V, Cella P, Ferrari A, Pezzetti F, Signorelli $\mathrm{C}$ and Getahun $\mathrm{H}$ : Assessing the impact of defining a global priority research agenda to address HIV-associated tuberculosis. Trop Med Int Health 21: 1420-1427, 2016.

8. Cardona PJ: The progress of therapeutic vaccination with regard to tuberculosis. Front Microbiol 7: 1536, 2016.

9. Bhargava S, Choubey S and Mishra S: Vaccines against tuberculosis: A review. Indian J Tuberc 63: 13-18, 2016.

10. Schnepp BC and Johnson PR: Vector-mediated in vivo antibody expression. Microbiol Spectr 2: AID-0016-2014, 2014.

11. Yang E, Wang F, Xu Y, Wang H, Hu Y, Shen H and Chen ZW: A lentiviral vector-based therapeutic vaccine encoding Ag85B-Rv3425 potently increases resistance to acute tuberculosis infection in mice. Acta Biochim Biophys Sin (Shanghai) 47: 588-596, 2015.

12. Dheda K, Barry CE III and Maartens G: Tuberculosis. Lancet 387 : 1211-1226, 2016.

13. Yang E, Gu J, Wang F, Wang $\mathrm{H}$, Shen $\mathrm{H}$ and Chen ZW: Recombinant BCG prime and PPE protein boost provides potent protection against acute Mycobacterium tuberculosis infection in mice. Microb Pathog 93: 1-7, 2016.

14. Wang J, Qie Y, Zhang H, Zhu B, Xu Y, Liu W, Chen J and Wang H: PPE protein (Rv3425) from DNA segment RD11 of Mycobacterium tuberculosis: A novel immunodominant antigen of Mycobacterium tuberculosis induces humoral and cellular immune responses in mice. Microbiol Immunol 52: 224-230, 2008.

15. Wang J, Qie Y, Liu W and Wang H: Protective efficacy of a recombinant BCG secreting antigen $85 \mathrm{~B} / \mathrm{Rv} 3425$ fusion protein against Mycobacterium tuberculosis infection in mice. Hum Vaccin Immunother 8: 1869-1874, 2012. 
16. Yang E, Lu Y, Xu Y, Liang Q, Wang C, Wang $\mathrm{H}$ and Shen $\mathrm{H}$ : Recombinant BCG coexpressing Ag85B, ESAT-6 and Rv3620c elicits specific Th1 immune responses in C57BL/6 mice. Microb Pathog 69-70: 53-59, 2014.

17. Shen H, Wang Y, Chen CY, Frencher J, Huang D, Yang E, Ryan-Payseur B and Chen ZW: Th17-related cytokines contribute to recall-like expansion/effector function of HMBPP-specific V 2 V8 2 T cells after Mycobacterium tuberculosis infection or vaccination. Eur J Immunol 45: 442-451, 2015.

18. Shen H, Wang C, Yang E, Xu Y, Liu W, Yan J, Wang F and Wang H: Novel recombinant BCG coexpressing Ag85B, ESAT-6 and mouse TNF-alpha induces significantly enhanced cellular immune and antibody responses in C57BL/6 mice. Microbiol Immunol 54: 435-441, 2010.
19. Kim JS, Kim WS, Choi HG, Jang B, Lee K, Park JH, Kim HJ, Cho SN and Shin SJ: Mycobacterium tuberculosis RpfB drives Th1-type T cell immunity via a TLR4-dependent activation of dendritic cells. J Leukoc Biol 94: 733-749, 2013.

20. Pontali E, Sotgiu G, Centis R, D'Ambrosio L, Spanevello A and Migliori GB: Management of drug resistantTB in patients with HIV co-infection. Expert Opin Pharmacother 16: 2737-2750, 2015.

21. Abate G and Hoft DF: Immunotherapy for tuberculosis: Future prospects. Immunotargets Ther 5: 37-45, 2016.

22. Palavecino CE, Céspedes PF, Gómez RS, Kalergis AM and Bueno SM: Immunization with a recombinant bacillus Calmette-Guerin strain confers protective Th1 immunity against the human metapneumovirus. J Immunol 192: 214-223, 2014. 